\title{
Splash plasma channels produced by picosecond laser pulses in argon gas for laser wakefield acceleration
}

\author{
Y. Mizuta, ${ }^{1}$ T. Hosokai, ${ }^{2,3, *}$ S. Masuda, ${ }^{2,3}$ A. Zhidkov, ${ }^{2,3}$ K. Makito, ${ }^{1}$ N. Nakanii, ${ }^{2,3}$ S. Kajino, ${ }^{1}$ A. Nishida, ${ }^{1}$ \\ M. Kando, ${ }^{4}$ M. Mori, ${ }^{4}$ H. Kotaki, ${ }^{4}$ Y. Hayashi, ${ }^{4}$ S. V. Bulanov, ${ }^{4,5}$ and R. Kodama ${ }^{1,2,3}$ \\ ${ }^{1}$ Graduate School of Engineering, Osaka University, 2-1 Yamadaoka, Suita, Osaka 565-0871, Japan \\ ${ }^{2}$ Photon Pioneers Center, Osaka University, 2-8 Yamadaoka, Suita, Osaka 565-0871, Japan \\ ${ }^{3}$ Japan Science and Technology Agency (JST), CREST, 2-8 Yamadaoka, Suita, Osaka 565-0871, Japan \\ ${ }^{4}$ Kansai Photon Science Institute, JAEA, Umemidai 8-1, Kizu, Kyoto 619-0215, Japan \\ ${ }^{5}$ Prokhorov General Physics Institute, Russian Academy of Sciences, Moscow 119991, Russia
}

(Received 23 May 2012; published 5 December 2012)

\begin{abstract}
Short-lived, $\sim 10 \mathrm{ps}$, deep plasma channels, with their lengths of $\sim 1 \mathrm{~mm}$ and diameters of $\sim 20 \mu \mathrm{m}$, are observed and characterized in Ar gas jets irradiated by moderate intensity, $\sim 10^{15-16} \mathrm{~W} / \mathrm{cm}^{2}$, laser pulses with a duration from subpicosecond to several picoseconds. The channels, upon 2D particle-in-cell simulations including ionization, fit well in the guiding of high intensity femtosecond laser pulses and, therefore, in laser wakefield acceleration with a controllable electron self-injection.
\end{abstract}

DOI: 10.1103/PhysRevSTAB.15.121301

PACS numbers: 52.38.Kd, 41.75.Jv, 52.38.Hb, 52.25.Jm

\section{INTRODUCTION}

Recent notable progress in the elaboration of laserdriven electron acceleration [1-3] with internal particle injections (the self-injection) in the acceleration phase of the laser pulse wake has brought up the problem of developing full-optical accelerators with controllable characteristics of accelerated particles. Primarily, such accelerators could make a basis of high-temporal and high-spatial resolution imaging systems [4] as well as the first stage in the ultrahigh energy multistage acceleration technique [5]. However, despite the high stability of gas jets and laser pulses, the laser wakefield acceleration (LWFA) technique gives only accelerated electrons which do not constitute a practical beam: the best electron energy spread $\sim 10 \%$, shot by shot energy and charge fluctuations $\sim$ by factors 2-3 and 10-100 correspondingly, pointing stability missing. The electron acceleration by laser wakefields may remain as only a sample of the laser plasma interaction if ways for the stable beam generation will not be found. One of the most serious sources of fluctuation is laser prepulses in the picosecond range $[5,6]$ and the amplified spontaneous emission [3]. Fluctuations induced by laser prepulses could be eliminated by artificial preplasma such as plasma channels with controlled parameters.

The use of plasma channels in the laser wakefield acceleration has been shown to provide a controllable self-injection that results in production of higher quality

\footnotetext{
*hosokai@ppc.osaka-u.ac.jp
}

Published by the American Physical Society under the terms of the Creative Commons Attribution 3.0 License. Further distribution of this work must maintain attribution to the author(s) and the published article's title, journal citation, and DOI. electron bunches [1-3,5-7]. Moreover, the longer guiding of the laser pulses in a plasma channel leads to a higher energy of accelerated electrons. There are several ways of making plasma channels suitable for the laser guiding: laser ablation [8], capillary discharges [9], and laserinduced plasma [5,10-13]. The last mentioned, being the full optical, seems to be more preferable for developing a jitter-free pump and probe system. The past decade there were several notable works on the creation of laser-induced plasma channels $[10,11]$. First, the collisional heating of plasma by long, $\sim$ ns, laser pulses has been used: preheated electrons move transversely from the vicinity irradiated by a laser pulse dragging ions out and, therefore, creating a plasma channel. This process usually takes a time order of $\sim 1 \mathrm{~ns}$; after $\sim$ hundreds of ns the channel disappears due to the retrograde waves. Higher intensity femtosecond laser pulses have also been used [12,13]. However, the mechanism of channel formation was similar and took time also the order of a nanosecond. In this case, after irradiation by a femtosecond laser pulse, energetic electrons are rapidly evacuated by the strong ponderomotive force charging the plasma because plasma ions are still immovable. Coulomb repulsion and the remaining electrons, which are heated in collisions, drag ions out. Nevertheless, if the pulse duration is longer, the order of a picosecond, the scenario of channel formation may become different.

It is clear that a real channel can be formed due to an ion displacement. The velocity of cold ions dragged by hot electrons is $v_{\text {ion }}=\sqrt{z T_{e} / M_{\text {ion }}}$, where $z$ is the ion charge $T_{e}$ is the electron temperature, and $M_{\text {ion }}$ is the ion mass. Neglecting the gas pressure and following Ref. [14], one can get the following for the plasma density at the laser axis: $N \approx N_{0}\left(w_{0} / v_{\text {ion }} t\right)^{2}$, where $w_{0}$ is the pulse waist, and 
$N_{0}$ is the initial ion density. Setting the temperature of electrons equal approximately to the ponderomotive potential of the laser pulse, $\sim m c^{2} a_{0}^{2} / 2$ and $a_{0}=e E / m c \omega$, where $E$ is the strength of the laser pulse field, and assuming that the channel is formed when $N=0.5 N_{0}$, for the laser intensity $I \sim 10^{15} \mathrm{~W} / \mathrm{cm}^{2}$ and $\lambda \sim 0.8 \mu \mathrm{m}$, one can get the necessary duration of the pulse about $\tau \sim 0.5 \mathrm{ps}$. That means a plasma channel can be formed with the use of a picosecond laser pulse if its intensity is the order of $I \sim 10^{15} \mathrm{~W} / \mathrm{cm}^{2}$. Such channels can be called splashplasma channels because, due to the retarded motion of plasma, such splash-plasma channels can exist only for a short time.

The splashlike channels have been observed in Ref. [5] in helium gas jets in the presence of the external magnetic field. In helium gas jets, such channels had been very rarely observed without a magnetic field. In Ref. [5] the splashlike channels were formed by fluctuating laser prepulses with undefined intensity and duration. Therefore the parameters of the splashlike channels could not been characterized.

In this paper, we experimentally investigate the formation of the splash channels exploiting well-defined picosecond laser pulses in argon gas occurring even without external magnetic fields and quantitatively characterize the channels using the picosecond time-resolved interferometer. Such channels, we anticipate, can tolerate the laser prepulses without significant change in the initial plasma parameters and drastically reduce the fluctuations in parameters of accelerated electrons. To understand usability of the channels for the laser wakefield acceleration, we perform two-dimensional (2D3V) particle-in-cell (PIC) simulations, which include ionization, with the initial conditions fitting the measured plasma profiles. PIC simulations in $2 \mathrm{D}$, we believe, are enough to demonstrate the existence or the absence of extra diffraction of laser pulses owing to the optical field ionization and guiding ability of splash channels.

\section{EXPERIMENTAL SETUP}

The experiment was performed at JLITE-X $800 \mathrm{~nm}$ Ti:sapphire chirped pulse amplification (CPA) laser system at the JAEA-KPSI (Kansai Photon Science Institute, Japan Atomic Energy Agency) [15,16]. An outline of the experimental setup is given in Fig. 1. Laser pulses with an initial diameter $\sim 30 \mathrm{~mm}$ were focused with $f / 5.9$ offaxis parabolic mirror to the position of $\sim 200 \mu \mathrm{m}$ behind the front edge of the slit gas jet and height of $\sim 1.5 \mathrm{~mm}$ form the nozzle exit. The focal spot size was $\sim 16 \mu \mathrm{m}$ in the full width at $1 / e^{2}$ of maximum; the corresponding Rayleigh length is approximately $186 \mu \mathrm{m}$. The energies of laser pulses with different durations, including the purepulses part, were constant and equal to $90 \mathrm{~mJ}$ in this experiment.
To study effects of intensity of laser pulses on the channel formation, durations of laser pulses were varied by tuning the distance of the gratings in the pulse compressor. The pulse durations of $0.3,1.8$, and $6.0 \mathrm{ps}$ were chosen as typical values with different ponderomotive potentials.

The maximum intensity of main pulses, corresponding to the pulse duration of $0.3 \mathrm{ps}$ on the target, was estimated to be $I=3.5 \times 10^{16} \mathrm{~W} / \mathrm{cm}^{2}$. The middle intensity of the main pulses, corresponding to the pulse duration of $1.8 \mathrm{ps}$, was estimated to be $I=5.7 \times 10^{15} \mathrm{~W} / \mathrm{cm}^{2}$, and the minimal intensity of main pulses, corresponding to the pulse duration of $6.0 \mathrm{ps}$, was $I=1.7 \times 10^{15} \mathrm{~W} / \mathrm{cm}^{2}$. Amplified spontaneous emission (ASE) prepulses with duration $\sim 3.5 \mathrm{~ns}$ were monitored by a photodiode and a third-order cross correlator (Amplitude Technologies Co., Ltd, Sequoia). Their typical shape in $100 \mathrm{ps}$ before the main pulse is shown in Fig. 2. The intensity of ASE prepulses was almost constant for every duration of main pulses and was estimated to be $I \sim 2 \times 10^{11} \mathrm{~W} / \mathrm{cm}^{2}$.

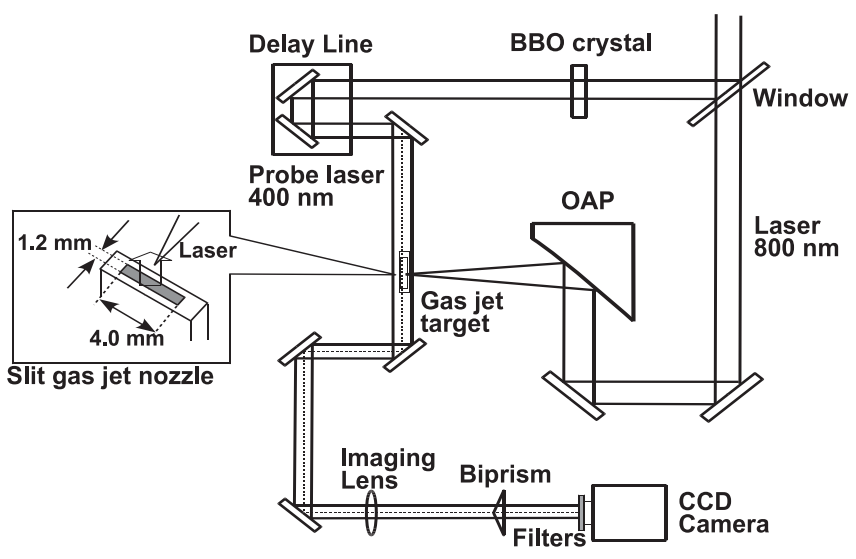

FIG. 1. Experimental setup.

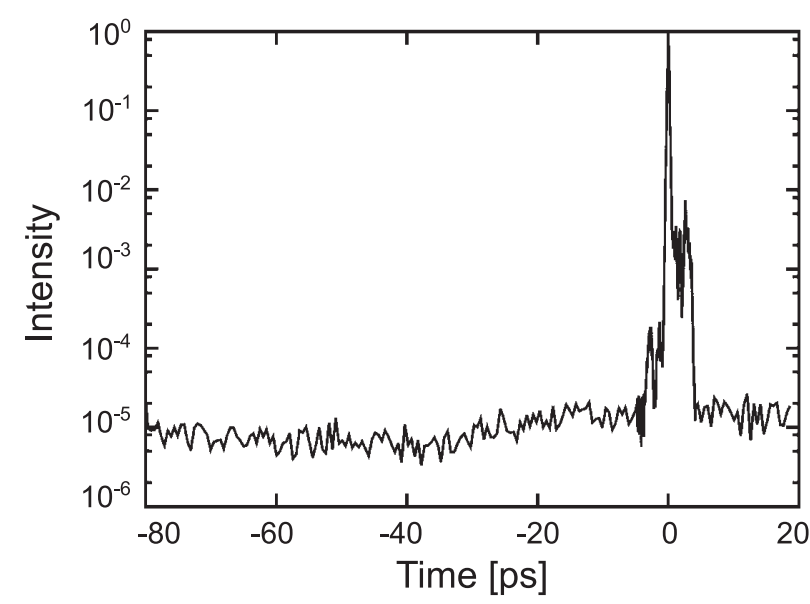

FIG. 2. Third-order autocorrelation trace of the driving laser. The ASE pedestal level is around $10^{-5}$. The pulse duration of the main pulse is $0.3 \mathrm{ps}$. 
Argon slit gas jets were used as a target. The dimension of the slit nozzle is $4 \times 1.2 \mathrm{~mm}^{2}$ as shown in the inset of Fig. 1. The slit gas jet was produced by the supersonic slit nozzle of Mach number 5 for argon gas, which is driven by a fast pulsed valve (Smartshell Co., Ltd A6 series). The uniform density distributions with the sharp boundary of the slit gas jet near the exit were characterized by interferometry with the computed tomography technique [7]. The neutral gas density at the height of $1.5 \mathrm{~mm}$ from the nozzle exit is estimated to be $N_{0}=1.5 \times 10^{19} \mathrm{~cm}^{-3}$ for the stagnation presser of the nozzle of $2.0 \mathrm{MPa}$.

In order to observe the formation of plasma channels, we used a time-resolved interferometer. Measurements were performed in the one-shot technique. As shown in Fig. 1, part of the main laser pulse carrying about $4 \%$ energy of the main pulse was split by a $4 \mu \mathrm{m}$ thickness window (National Photocolor Co., Ltd: Pellicle). It was delivered into the target region as a probe beam for the plasma diagnosis in the direction of $90^{\circ}$ from the laser propagation axis. For the interferometry of the plasma, a biprism was installed on the probe laser axis behind the imaging lens. The wavelength of the probe laser pulses was $400 \mathrm{~nm}$, which was produced with a $2 \mathrm{~mm}$-thickness $\mathrm{BBO}$ (barium metaborate: $\mathrm{BaB}_{2} \mathrm{O}_{4}$ ) crystal. The interferograms are imaged on a 16 bit charge coupled device (CCD) camera. A band-pass filter $(\Delta \lambda=10 \mathrm{~nm}$ at $\lambda=400 \mathrm{~nm})$ was put in front of the CCD to cut the plasma light. Synchronization of the probe beam with the evolving plasma was adjusted by changing of the length of the optical path on the delay line. Spatial resolution of the measurement was $5 \mu \mathrm{m}$; temporal resolution of the measurement was the same as the pulse duration of the probe beams depending on the main pulse.

\section{SPLASH-PLASMA CHANNELS}

Probing-pulse-time-integrated interferograms of traces which occur during the propagation of a CPA laser pulse through argon gas jet target are shown in Figs. 3-5 for laser pulses of $0.3,1.8$, and $6 \mathrm{ps}$, respectively. Several time delays between the main pulse and probing pulse, 1, 3, 6, and $9 \mathrm{ps}$, were chosen to illustrate the most important stages of channel formation and relaxation. (In all figures, laser pulses come from the left side.) Corresponding electron density distributions are shown in Figs. 6-8. The electron density distributions were calculated from the phase shift. Some cases required a small postprocessing on the phase-shift discontinuity. IDEA code was used to obtain a phase-shift map [17]. Then, the fast Fourier transform technique was used to retrieve the plasma-imposed phase shift [18]. The phase-shift map was processed then with an Abel inversion algorithm [19,20] assuming the cylindrical symmetry of the channels. Restored transverse electron density distributions at different positions, A, B, C as in Fig. 6(d), in Fig. 7(d), and in Fig. 8(d), are shown in Figs. 9-11, respectively.

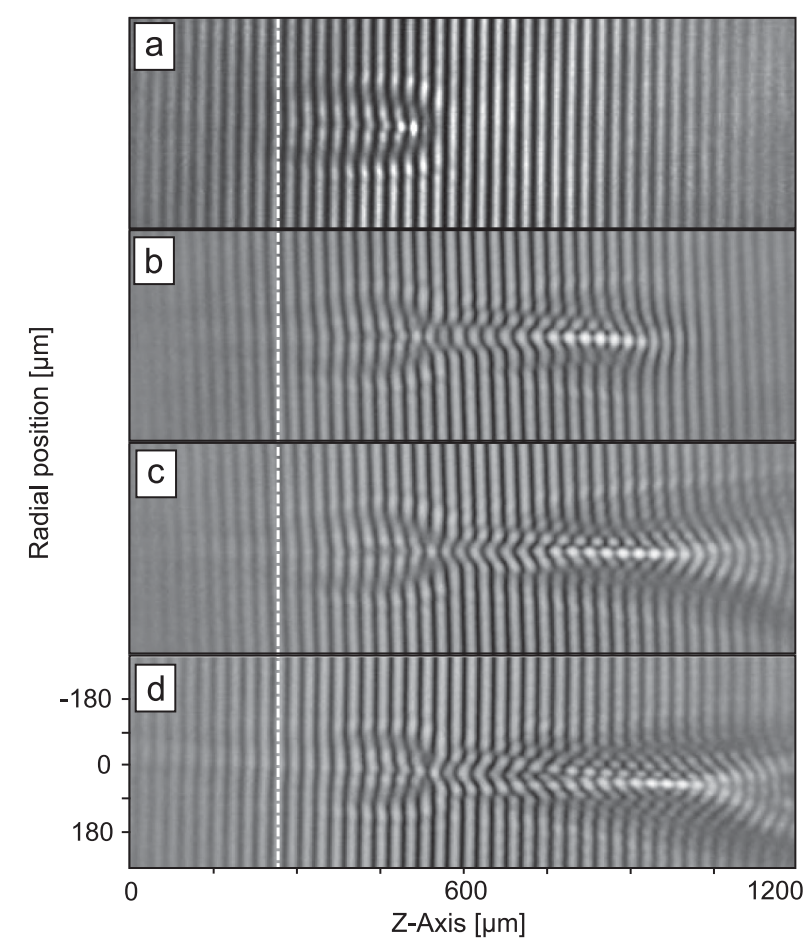

FIG. 3. Interferograms of the plasma channel at the laser power $P=0.3 \mathrm{TW}$ (the pulse energy $90 \mathrm{~mJ}$ and pulse duration $0.3 \mathrm{ps}$ ) and $1.5 \times 10^{19} \mathrm{~cm}^{-3}$ gas density at different times. Parts (a)-(d) show the interferograms at different time delays: (a) 1 ps, (b) 3 ps, (c) 5 ps, (d) 9 ps.

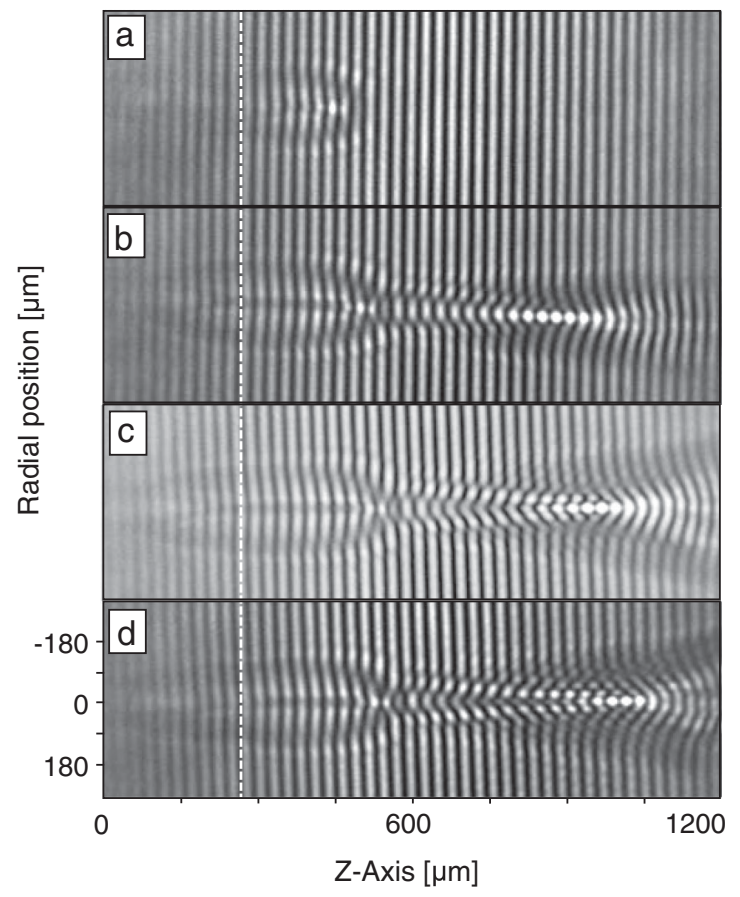

FIG. 4. Interferograms of the plasma channel for the laser power $P=50 \mathrm{GW}$ (the pulse energy of $90 \mathrm{~mJ}$ and pulse duration of $1.8 \mathrm{ps}$ ) and $1.5 \times 10^{19} \mathrm{~cm}^{-3}$ gas density at different times. Parts (a)-(d) show the interferograms at different time delays: (a) 1 ps, (b) 3 ps, (c) 5 ps, (d) 9 ps. 


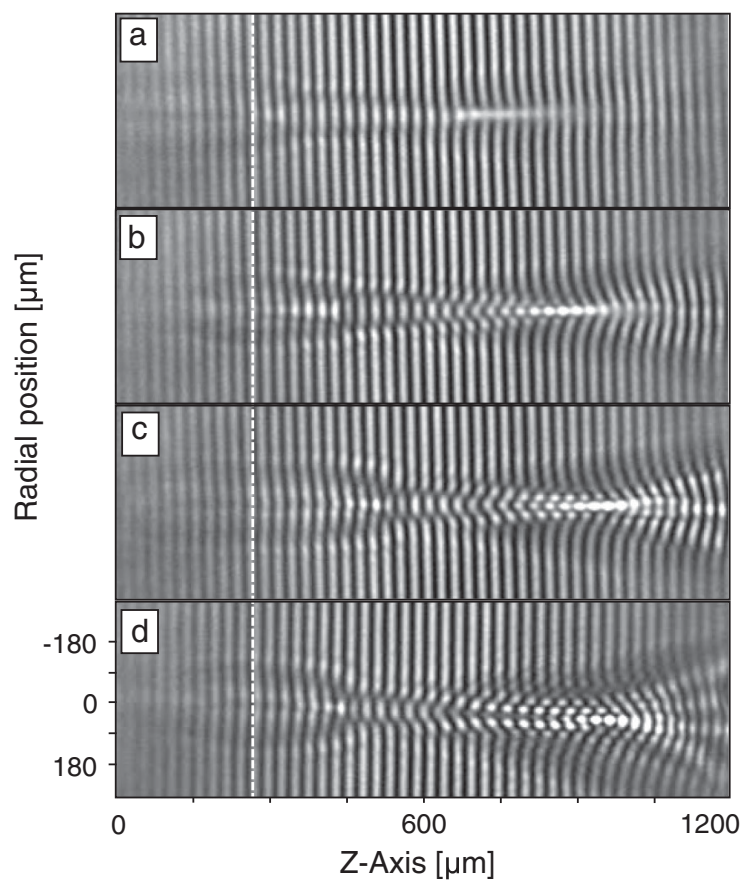

FIG. 5. Interferograms of the plasma channel for the laser power $P=15 \mathrm{GW}$ (the pulse energy of $90 \mathrm{~mJ}$ and pulse duration of $6 \mathrm{ps}$ ) and $1.5 \times 10^{19} \mathrm{~cm}^{-3}$ gas density at different times. Parts (a)-(d) show the interferograms at different delays: (a) $1 \mathrm{ps}$, (b) $3 \mathrm{ps}$, (c) $5 \mathrm{ps}$, (d) 9 ps.

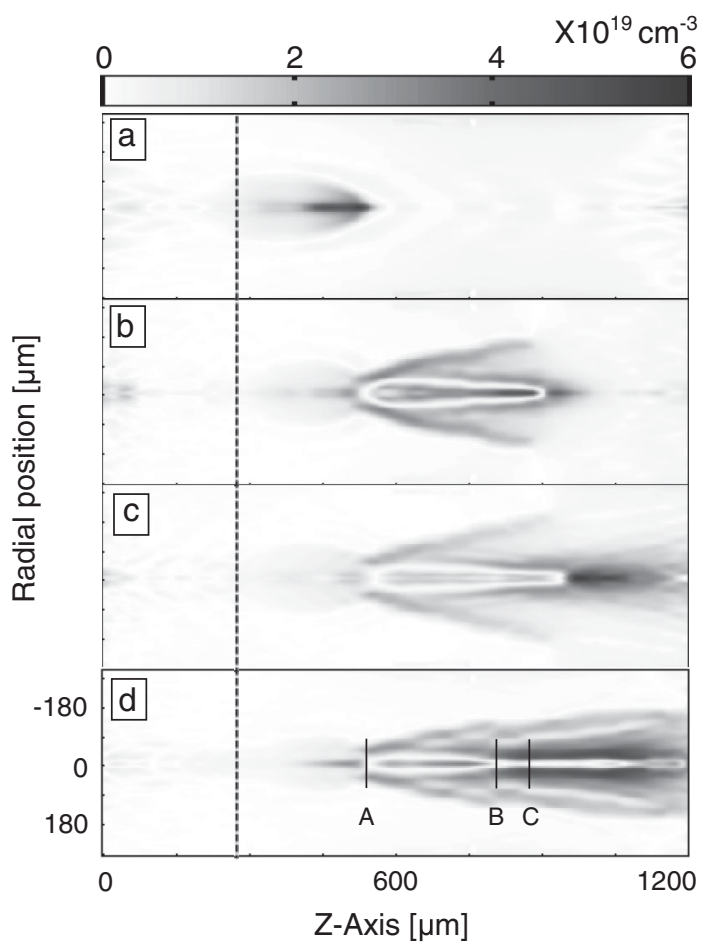

FIG. 6. Restored electron density distribution of the plasma channel for $P=0.3 \mathrm{TW}$ (the pulse energy $90 \mathrm{~mJ}$ and pulse duration $0.3 \mathrm{ps}$ ) and $1.5 \times 10^{19} \mathrm{~cm}^{-3}$ gas density at different times. Parts (a)-(d) show the interferograms at different time delays: (a) 1 ps, (b) 3 ps, (c) 5 ps, (d) 9 ps.

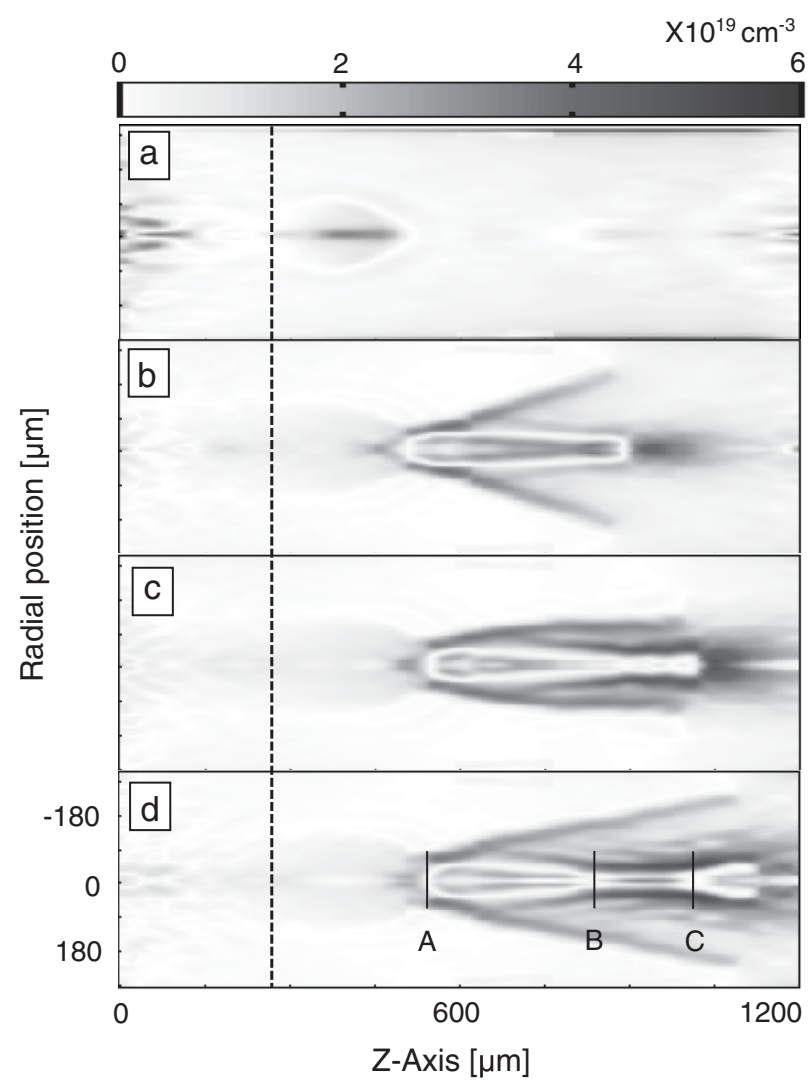

FIG. 7. Restored electron density distribution of the plasma channels for the laser power $P=50 \mathrm{GW}$ (the pulse energy of $90 \mathrm{~mJ}$ and pulse duration of $1.8 \mathrm{ps}$ ) and $N_{0}=1.5 \times 10^{19} \mathrm{~cm}^{-3}$ gas density at different times. Parts (a)-(d) show the interferograms at different time delays: (a) 1 ps, (b) 3 ps, (c) 5 ps, (d) 9 ps.

One can see the formation of channel structures in all cases for durations from 0.3 to 6 ps. However, the structure essentially depends on the pulse duration as seen in the transverse cross section of the density profile (see Figs. 9-11). The differences are demonstrated in Fig. 12. At the pulse duration of $0.3 \mathrm{ps}$, the length of the plasma channel is as long as $600 \mu \mathrm{m}$ as shown in Fig. 12(a). It is approximately 3 times longer than the Raleigh length. The length decreases with pulse duration down to $400 \mu \mathrm{m}$ for 6 ps pulse duration. The opposite dependency is observed for the channel diameter; being about $15 \mu \mathrm{m}$ in the case of $0.3 \mathrm{ps}$ pulse duration, it increases up to $37 \mu \mathrm{m}$ for 6 ps duration pulses as shown in Fig. 12(b). The channel depth (the ratio of maximal and minimal electron density) is not monotonic, Fig. 12(c). It falls from $\sim 50$ to 25 when the duration increases form 0.3 to $1.7 \mathrm{ps}$. Then it increases again to the factor of 35 . We attribute this to decreasing of ponderomotive force with laser intensity decrease, as $\tau^{-1}$, where $\tau$ is the pulse duration, and to the growing effect of elastic collisions which result in the electron heating at a longer pulse duration. Figure 13 gives insight into the difference between the channels formed by shorter and longer laser pulses after 9 ps of plasma relaxation. For the highest 


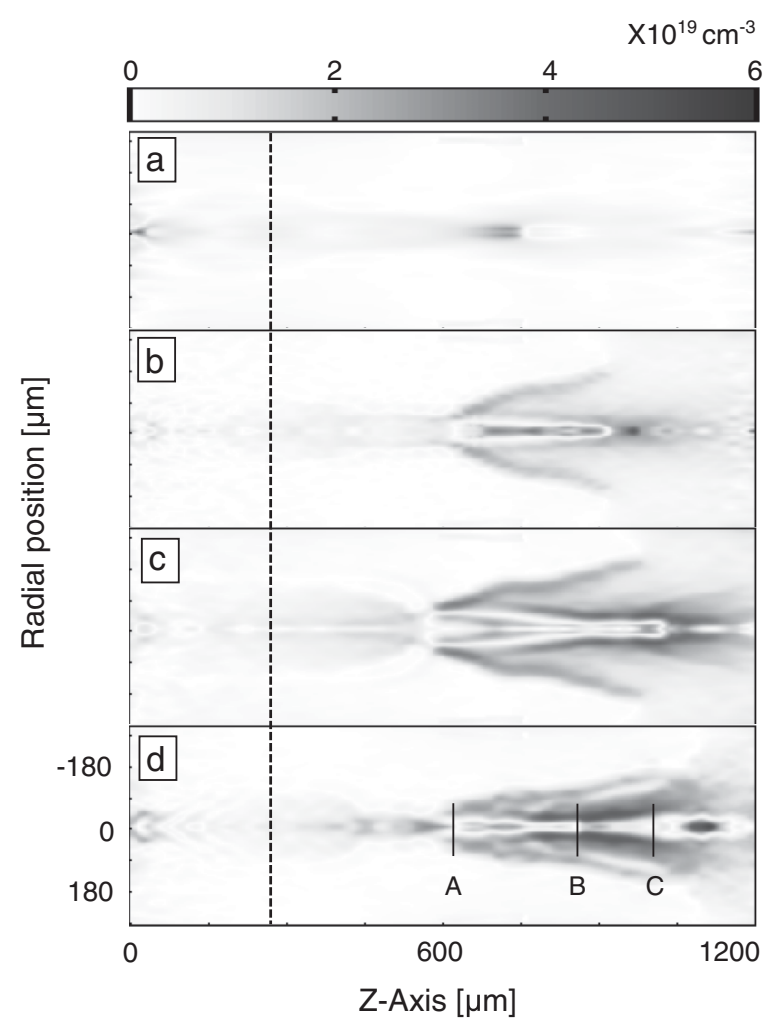

FIG. 8. Restored electron density distribution of the plasma channels for the laser power $P=15 \mathrm{GW}$ (the pulse energy of $90 \mathrm{~mJ}$ and pulse duration of $6 \mathrm{ps}$ ) and $1.5 \times 10^{19} \mathrm{~cm}^{-3}$ gas density at different times. Parts (a)-(d) show the interferograms at different delays: (a) 1 ps, (b) 3 ps, (c) 5 ps, (d) 9 ps.

intensity (the shortest pulse of $0.3 \mathrm{ps}$ ), the ponderomotive potential, and the hot electron energy, is as $\sim 6 \mathrm{keV}$. This energy is sufficient for ion drag in 1 ps to a distance $\sim 10 \mu \mathrm{m}$. For the lowest intensity (the longest pulse of $6 \mathrm{ps}$ ), the ponderomotive potential is $\sim 150 \mathrm{eV}$ and is not enough for direct ion drag. However, the elastic collisions may result in a rapid growth of electron temperature up to $\sim 1-2 \mathrm{keV}$ resulting in the ion motion. The pulse duration necessary for the collision heating can be estimated with the well-known elastic collision frequency: $\nu \sim 5 \times$ $10^{-5} Z^{2} N_{i} / T_{e}^{3 / 2}$ [14]. For $Z \sim 3, N_{i} \sim 3 \times 10^{19} \mathrm{~cm}^{-3}$, and $T_{e} \sim 100 \mathrm{eV}$ it is approximately equal to $\nu^{-1} \sim 0.1 \mathrm{ps}$, while at $T_{e} \sim 1 \mathrm{keV}, \tilde{\nu}^{1} \sim 2 \mathrm{ps}$. That means for the efficient heating the pulse duration should be longer than a picosecond [14]. In all cases the deep channel structure still exists. However, the change of electron heating mechanism is seen in the dependencies presented in Figs. 12 and 13. The shortest pulse makes a thinner and deeper channel, while the channel structures for 1.7 and 6 ps laser pulses are quite similar.

However, all these measurements were performed for the electron density. The ion density was not measured in the present experiments. Even though it is clear that

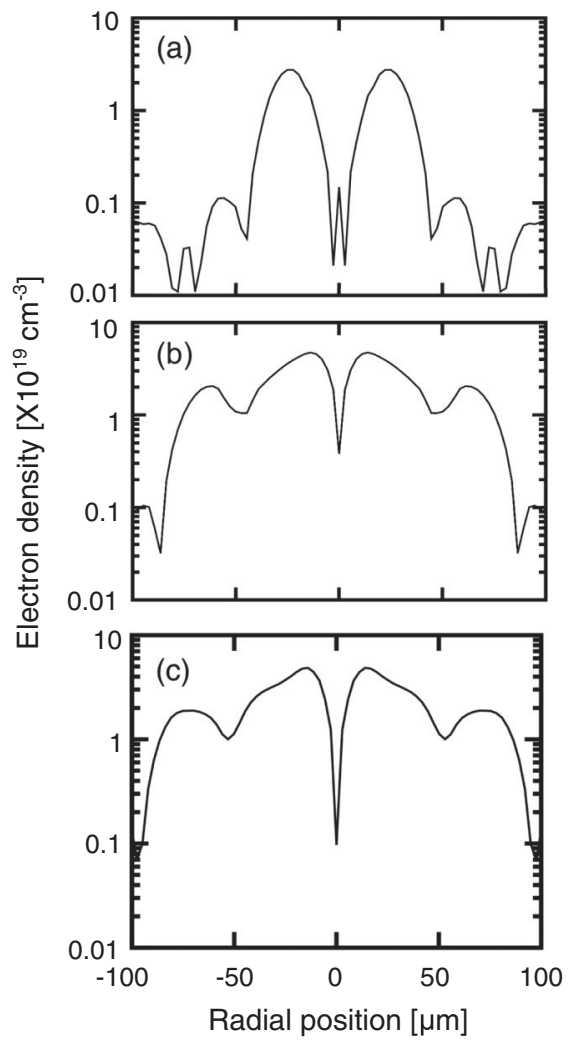

FIG. 9. Transverse cross section of density profiles at different positions: A, B, and $\mathrm{C}$ as in Fig. 6(d).

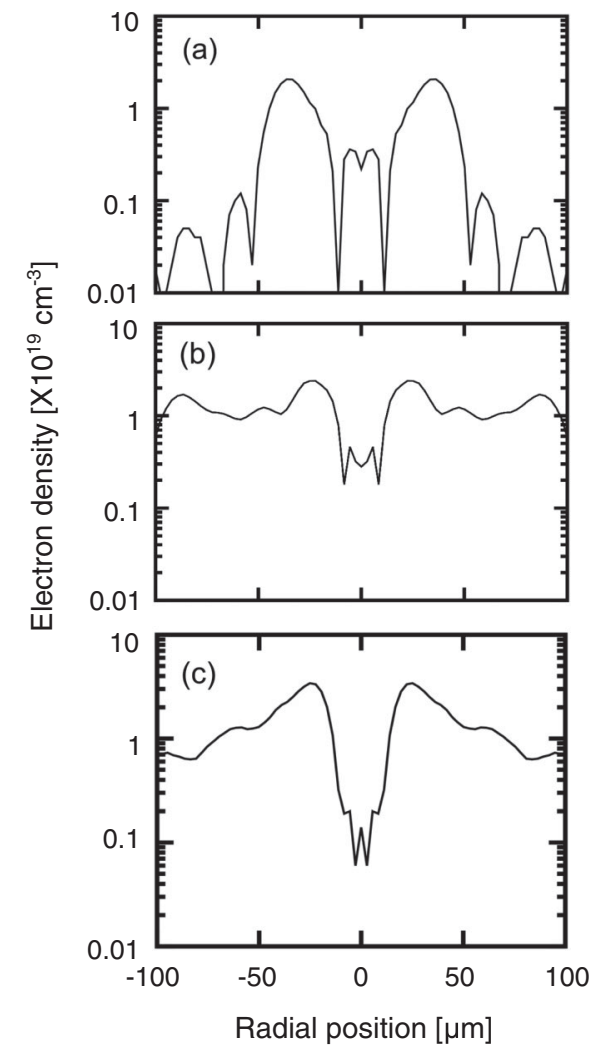

FIG. 10. Transverse electron density distribution at different positions: A, B, and $\mathrm{C}$ as in Fig. 7(d). 


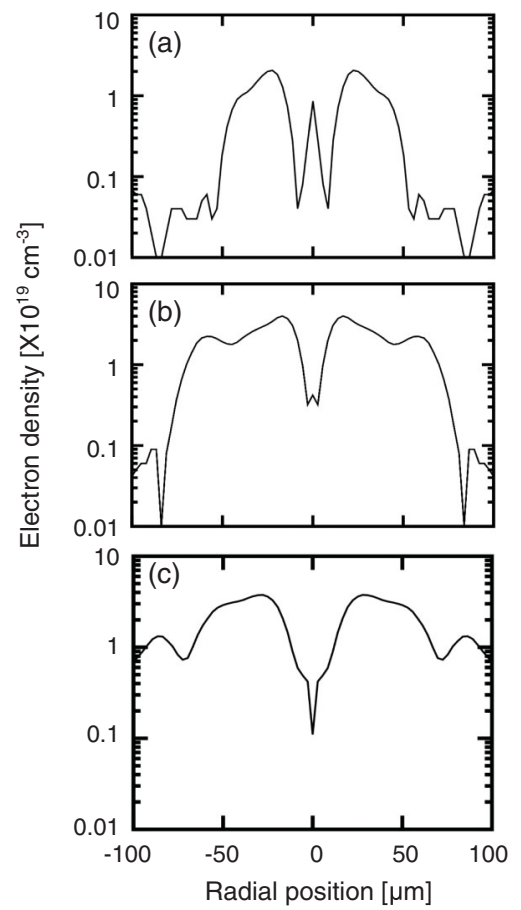

FIG. 11. Transverse electron density distribution at different positions: A, B, and $\mathrm{C}$ as in Fig. 8(d).
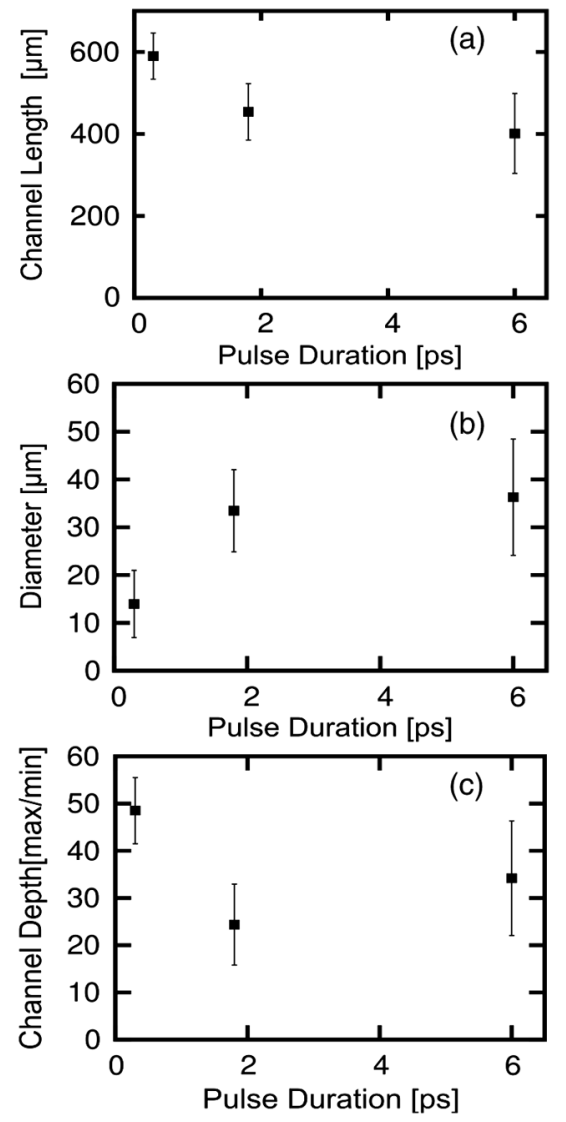

FIG. 12. Plasma channel parameters at different pulse durations (0.3, 1.8, and 6 ps). (a) Channel length, (b) channel diameter, (c) channel depth (maximal density/minimal density).

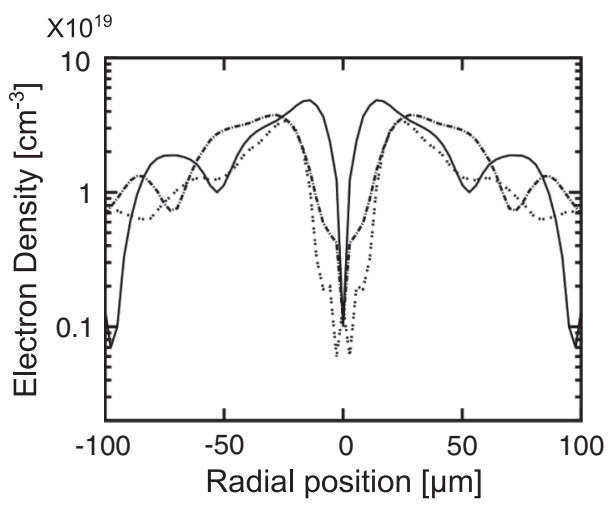

FIG. 13. Transverse density profiles at different pulse durations. Solid line: 0.3 ps [Fig. 6(c)], dotted line: 1.8 ps [Fig. 7(c)], dashed line: 6 ps [Fig. 8(c)].

observed channel structures cannot be a result of atomic physics only, we have to prove this. In Fig. 14 the dynamics of transverse density distribution at the channel entrance and the channel exit are given for pulse duration of 0.3 ps. One can see that in the beginning the density profile is quite usual: its maximum reaches in the channel axis. In the case of the $0.3 \mathrm{ps}$ laser pulse, the ionization is mostly due to the optical field ionization. After the laser pulse past in cold plasma, the electron density should decrease due to the three body recombination [14]. The time of the recombination is approximately $\tau_{R} \approx 1.6 \times$ ${ }^{10}{ }^{26} T_{e}^{9 / 2} / z^{3} N_{e}^{2}$, where $T_{e}$ is the electron temperature in
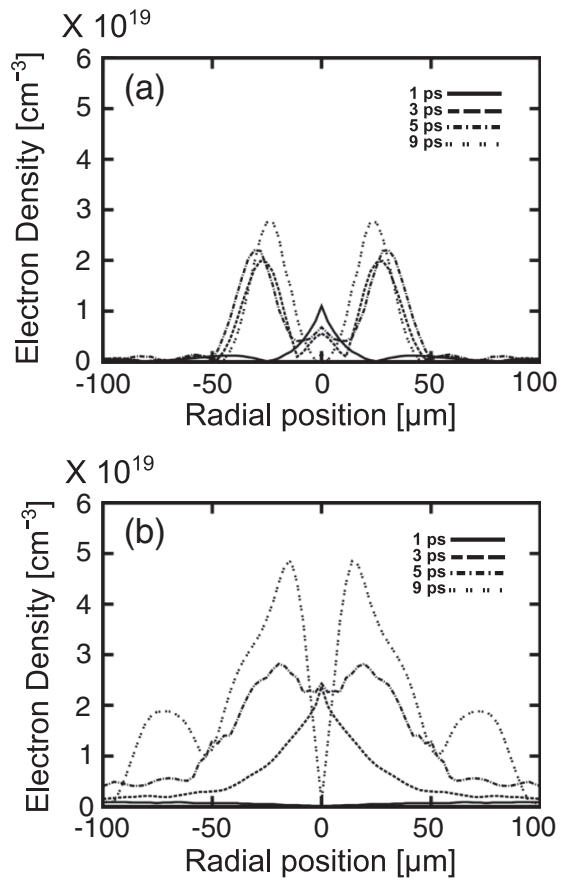

FIG. 14. Transverse density profiles of the plasma channels at the pulse duration of 0.3 ps for different times. (a) Electron density distribution at the entrance of the plasma channel. (b) Electron density distribution at the channel exit. 
$\mathrm{eV}, z$ is the ion charge, and $N_{e}$ is the electron density in $\mathrm{cm}^{-3}$. For the electron density from our measurements and $z=1$, the recombination time exceeds $\sim 1$ ps for the electron temperature of only $1 \mathrm{eV}$, and it exceeds $30 \mathrm{~ns}$ for $T_{e}=10 \mathrm{eV}$. Therefore the recombination in such a short time scale plays no role. Collisional ionization may result in the electron density profile. Using the Lotz approximation for ionization cross section, $\sigma_{\text {ion }} \approx$ $4 \times 10^{-14} \xi \ln (\varepsilon / I) / \varepsilon I\left[\mathrm{~cm}^{2}\right]$, where $\xi$ is the number of equivalent electrons in a shell, $\varepsilon$ is the electron energy in $\mathrm{eV}$, and $I$ is the ionization potential in $\mathrm{eV}$, one can get for the ionization time in $\operatorname{Ar}: \tau \sim\left(\sigma_{\text {ion }} v N_{e}\right)^{-1} \sim 0.3$ ps for $\varepsilon=100 \mathrm{eV}$. However, the collisional ionization cannot explain the reduction of electron density in the channel axis and the retreat wave at $t=9 \mathrm{ps}$ as seen in Fig. 14 . These estimations prove the ion motion took place with the formation of a splash-plasma channel.
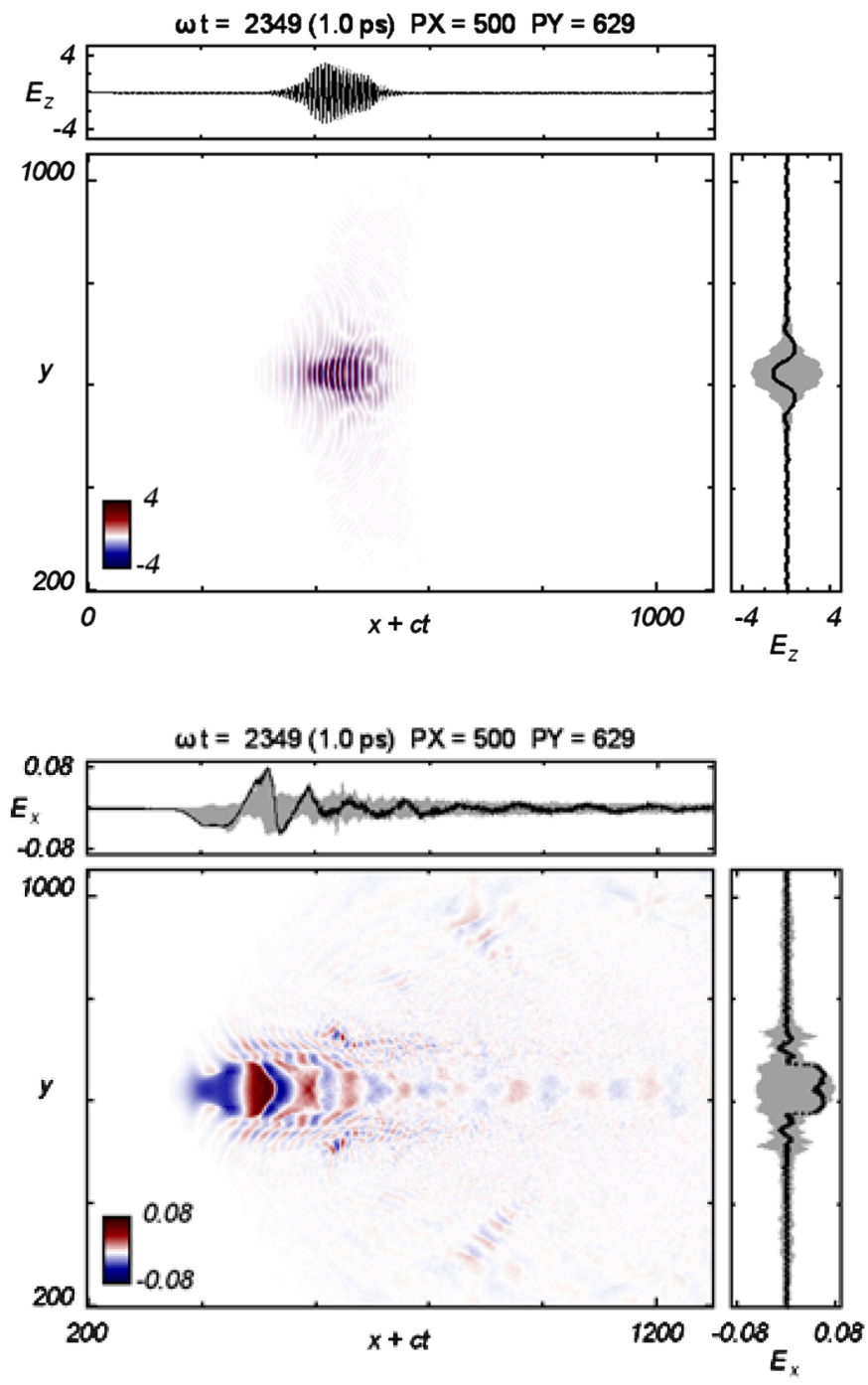

\section{PIC SIMULATION OF LWFA IN A SPLASH CHANNEL}

To estimate the usefulness of the splash channel for the laser wakefield acceleration, we perform 2D particle-incell simulation which included the optical field ionization of plasma. We use the variable particle weight approach [21] to calculate plasma ionization. The method is based on the solution of balance equations for density and momentum $[14,21]$ :

$$
d N_{i} / d t=S_{i-1} N_{i-1}, \quad N_{D}=\sum_{i=0} N_{i}, \quad d \vec{P} / d t=0,
$$

where $S_{i}$ is the ionization rates for the $i$ th ion, $N_{e}$ is the electron density, $N_{D}$ is the gas density, and $P$ is the total momentum. The ionization rate can be written in the well known approximation used in Refs. [21-24]:
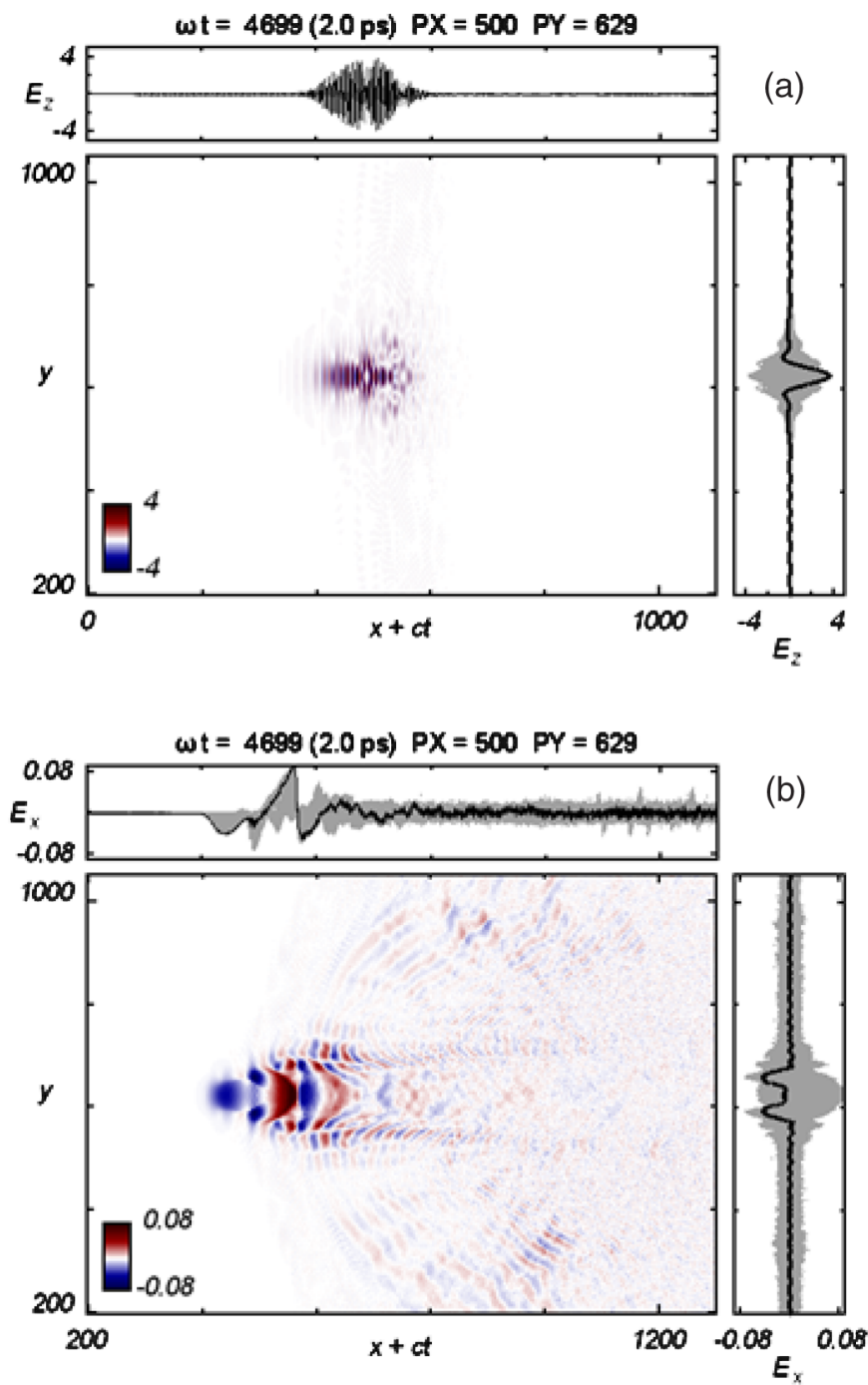

FIG. 15. Calculated laser and plasma field distributions: (a) Laser field distribution at different times, (b) Plasma field distributions at different times. 


$$
S_{k}=4 \omega_{A} g_{k}\left(\frac{I_{k}}{R y}\right)^{5 / 2} \frac{E_{A}}{E} \exp \left(-\frac{2}{3}\left(\frac{I_{k}}{R y}\right)^{3 / 2} \frac{E_{A}}{E}\right),
$$

where $\omega_{A}=m e^{4} / \hbar^{3}$ and $E_{A}=m^{2} e^{5} / \hbar^{4}$ the atomic frequency and atomic electric field strength; $R y=$ $m^{2} e^{4} / 2 \hbar^{2} ; I_{k}$ is the potential for ion with charge $k ; g_{k}$ is a factor $\sim 1 ; E$ is an external field strength. The energy and momentum conservation is achieved by introducing an atomic current, which provides the Ohmic heating equivalent to the power loss for the ionization: $\vec{j}_{A} \vec{E}=$ $\sum_{k=0} I_{k} S_{k} N_{k}$, to Maxwell equations. In the method, a charge growth is calculated from the balance equations as $\Delta Q_{M}= \pm e \Delta t V_{M} \sum_{k=0} S_{k} N_{k}$ and is redistributed over all particles in a kinetic cell $M$ with its volume $V_{M}$.

PIC simulations are performed for a linearly polarized laser pulse propagating in $\mathrm{Ar}$ under $1 / 2$ of the normal pressure or $N_{0}=1.5 \times 10^{19} \mathrm{~cm}^{-3}$, and for the laser, intensity $I=3 \times 10^{19} \mathrm{~W} / \mathrm{cm}^{2}, \lambda=0.8 \mu \mathrm{m}$, and $40 \mathrm{fs}$ full width at half maximum duration. The $s$-polarized laser pulse is numerically focused in the $16 \mu \mathrm{m}$ diameter in $150 \mu \mathrm{m}$ numerical focal length. The initial density profile is chosen to emulate the density profile from the experiment with 6 ps laser pulse, see Sec. III. While the channel diameter is kept constant at $D=37 \mu \mathrm{m}$, the channel depth is a parameter. In the PIC simulation we use the code FPLASER2D [22] exploiting the moving window technique. We use a $320 \mu \mathrm{m} \times 150 \mu \mathrm{m}$ window and $\lambda / 10$ spatial resolution; the kinetic cell is 2 times as large as the PIC one.

The laser and plasma field distributions for the deepest plasma channel are given in Fig. 15. They exhibit the typical field distribution for the plasma guiding: the strong self-focusing and well-structured pulse wake. The diffraction of the light through the thin channel border exists but it is not strong. Electron density distribution for two channels with different depth is shown in Figs. 16(a) and 16(b). One can see that the ionization of higher density plasma
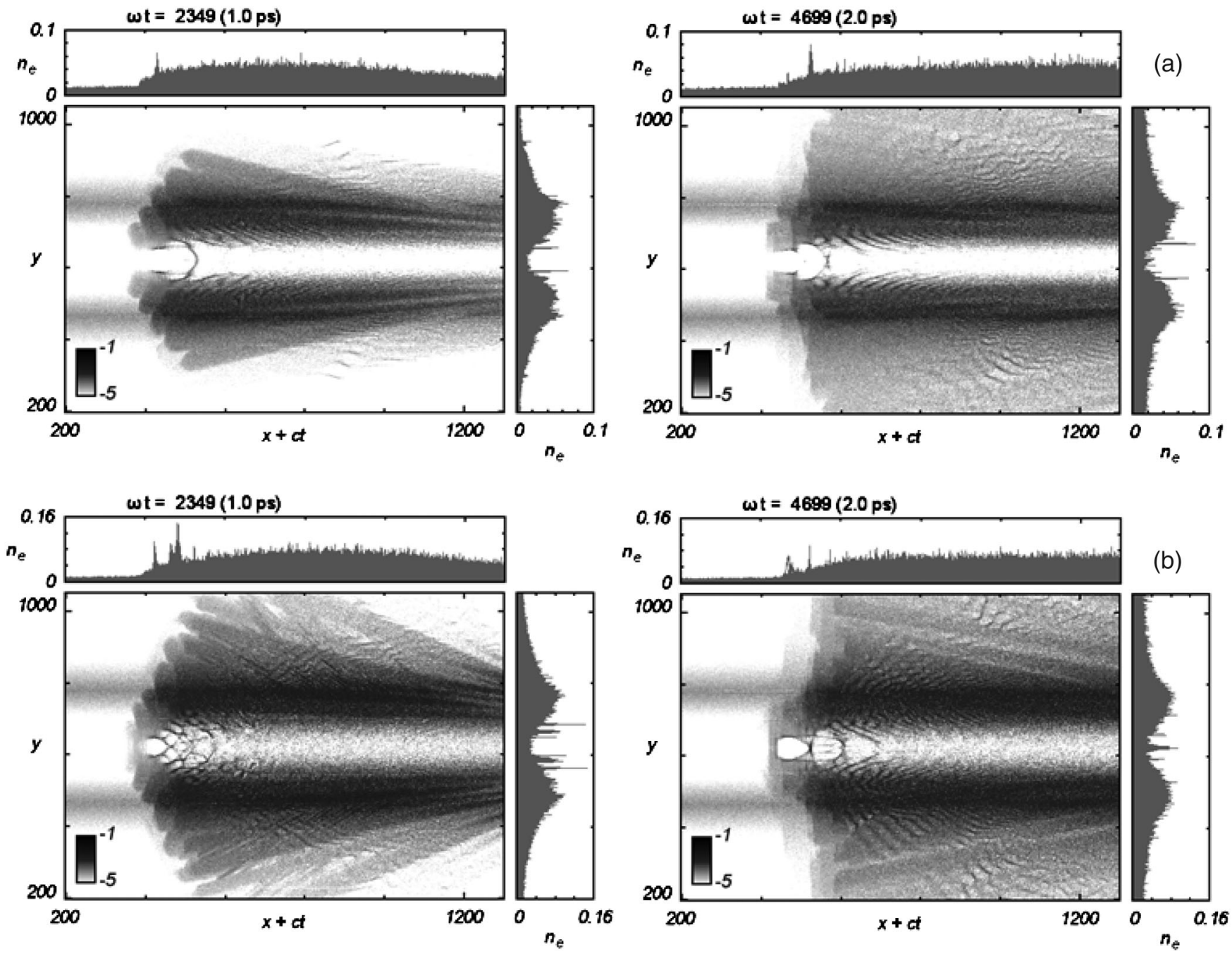

FIG. 16. Spatial distribution of electron density distribution in plasma channels with the different depths: (a) maximum electron density is $3 \times 10^{19} \mathrm{~cm}^{-3}$ and minimum density is $0.5 \times 10^{18} \mathrm{~cm}^{-3}$; (b) maximum electron density is $3 \times 10^{19} \mathrm{~cm}^{-3}$ and minimum density is $2.0 \times 10^{18} \mathrm{~cm}^{-3}$. 
periphery by the main laser pulse changes the electron density distribution in the splash channel. Therefore, the initial plasma structure cannot completely determine the electron self-injection and provide the controllable selfinjection at the long pulse guiding; the ionization effects have to be taken into account. Nevertheless, finally, the electron acceleration can be controlled by the splash channel structure.

In Fig. 17 spatial distribution of momenta of accelerated electrons for two different plasma channels are given. One can see that in the shallow channel the maximal energy of electrons is expectedly lower than that in the deepest
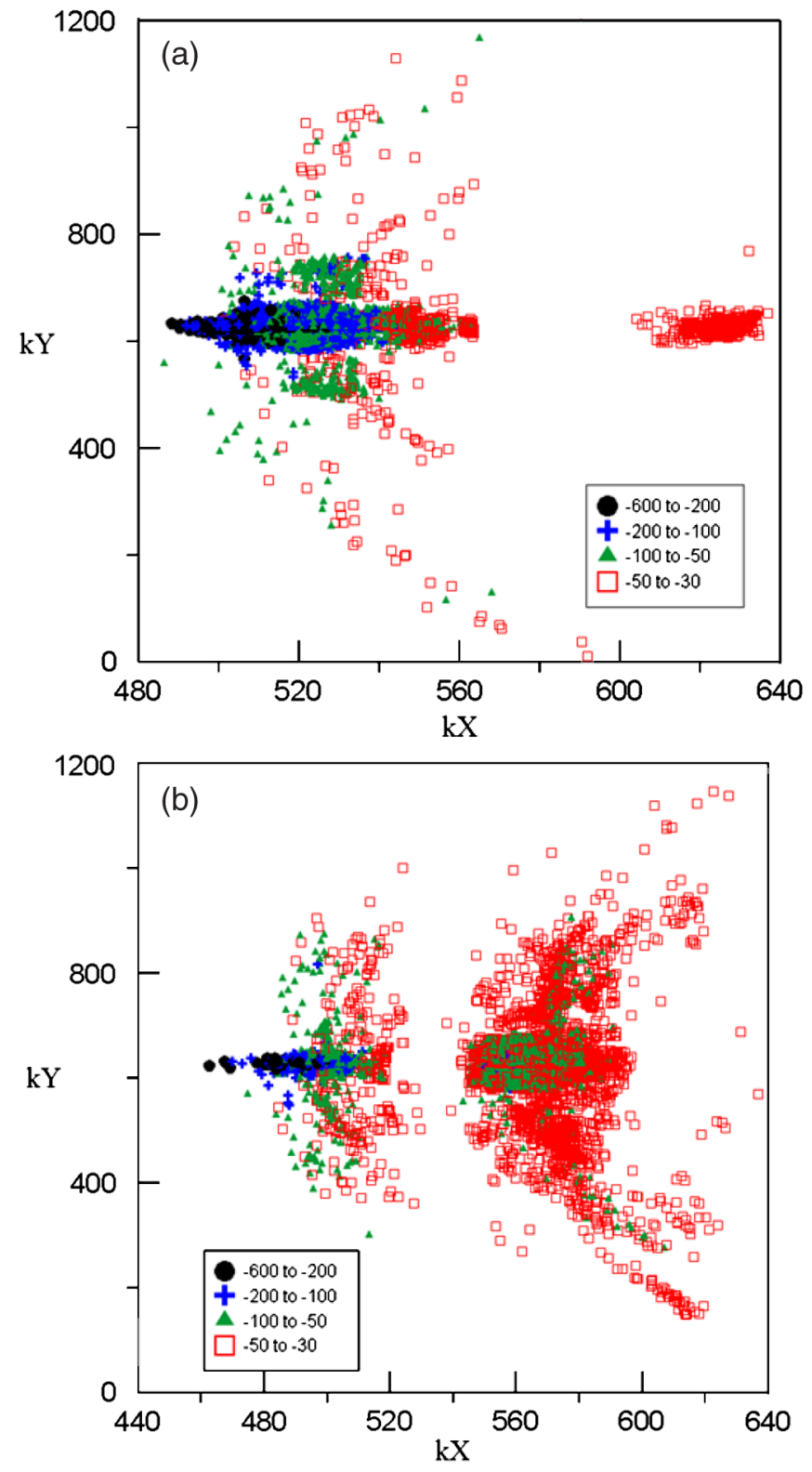

FIG. 17. Spatial distribution of accelerated electron momenta in two plasma channels: (a) minimal electron density is $0.5 \times$ $10^{18} \mathrm{~cm}^{-3}$; (b) minimal electron density is $2.0 \times 10^{18} \mathrm{~cm}^{-3}$. channel. The geometrical emittance and energy spread are also poor in the shallow channel. That means the formation of the splash channel may result in the quality of the electron beams and their total charge. The energy spread also depends on the channel depth: it decreases from $30 \%$ for the shallow splash channel down to $8 \%$ for the deep channel.

\section{CONCLUSION}

We have studied the process of formation and relaxation of short-lived plasma channels (splash channels), produced by picosecond laser pulses in argon gas jets. The quantitative characterization of splash-plasma channels has been performed with the use of the picosecond time-resolved interferometry. In the present experiments we could produce a splash channel with its length $\sim 600 \mu \mathrm{m}$, which is 3 times larger than the Rayleigh length. The channel diameter depends on the pulse duration and is about $15-30 \mu \mathrm{m}$; the minimal electron density in the channels is almost 2 orders lower than the maximal. The lifetime of the channels is about $10 \mathrm{ps}$. We can also accept that far longer channels with their lengths of the order of several mm can be created by this technique (see also Ref. [5]).

Upon use of two-dimensional particle-in-cell simulations which include the plasma ionization (only optical field ionization was taken into account), we have demonstrated the usefulness of splash channels for the laser wakefield acceleration. The quasimonoenergetic beams $(\Delta E / E<10 \%)$ with their stability and parameters determined by splash channel structure can be generated. Apparently, the splash channel structure cannot be essentially changed by the laser prepulse with the energy less than $90 \mathrm{~mJ}$. That supports such acceleration techniques as a basis for high-temporal and high-spatial resolution imaging systems as well as the first stage in the ultrahigh energy multistage laser acceleration.

\section{ACKNOWLEDGMENTS}

This work was supported by Core Research for Evolutional Science and Technology (CREST) of the Japan Science and Technology Agency (JST). It was also supported by the Global Centers of Excellence (GCOE) Program of the Japan Society for the Promotion of Science.

[1] J. Faure, Y. Glince, A. Pukhov, S. Kiselev, S. Gordienko, E. Lefebvre, J.-P. Rousseau, F. Burgy, and V. Malka, Nature (London) 431, 541 (2004); S. P. D. Mangles, C. D. Murphy, Z. Najmudin, A.G. R. Thomas, J. L. Collier, A. B. Dangor, E. J. Divall, P. S. Foster, J. G. Gallacher, C. J. Hooker, D. A. Jaroszyanski, A. J. Langley, W. B. Mori, P. A. Norreys, F. S. Tsung, R. Viskop, B. R. Walton, and K. Krushelnick, Nature (London) 431, 535 (2004); W.P. Leemans, B. Nagler, A. J. Gonsalves, C. Toth, K. Nakamura, C. G. R. Geddes, E. Esarey, 
C. B. Schroeder, and S.M. Hooker, Nat. Phys. 2, 692 (2006).

[2] C. G. R. Geddes, Cs. Toth, J. Van Tolborg, E. Esarey, C. B. Schroeder, D. L. Bruhwiler, C. Nieter, J. R. Cary, and W. P. Leemans, Nature (London) 431, 538 (2004); C. G. R. Geddes, Cs. Toth, J. van Tilborg, E. Esarey, C. B. Schroeder, C. Nieter, J. Cary, and W. P. Leemans. Phys. Plasmas 12, 056709 (2005).

[3] T. Hosokai, K. Kinoshita, A. Zhidkov, K. Nakamura, T. Watanabe, T. Ueda, H. Kotaki, M. Kando, K. Nakajima, and M. Uesaka, Phys. Rev. E 67, 036407 (2003); T. Hosoaki, K. Kinoshita, A. Zhidkov, K. Nakamura, H. Kotaki, M. Kando, K. Nakajima, and M. Uesaka, Plasma Phys. 11, L57 (2004).

[4] J. S. Kim, T. LaGrange, B. W. Reed, M. L. Taheri, M. R. Armstrong, W.E. King, N.D. Browning, and G. H. Campbell, Science 321, 1472 (2008).

[5] T. Hosokai, A. Zhidkov, A. Yamazaki, Y. Mizuta, M. Uesaka, and R. Kodama. Appl. Phys. Lett. 96, 121501 (2010).

[6] T. Hosokai, K. Kinoshita, A. Zhidkov, A. Maekawa, A. Yamazaki, and M. Uesaka, Phys. Rev. Lett. 97, 075004 (2006).

[7] T. Hosokai, K. Kinoshita, T. Ohkubo, A. Maekawa, M. Uesaka, A. Zhidkov, A. Yamazaki, H. Kotaki, M. Kando, K. Nakajima, S. V. Bulanov, P. Tomassini, A. Giulietti, and D. Giulietti, Phys. Rev. E 73, 036407 (2006); T. Ohkubo, S. V. Bulanov, A. G. Zhidkov, T. Esirkepov, J. Koga, M. Uesaka, and T. Tajima, Phys. Plasmas 13, 103101 (2006).

[8] Y. Kitagawa, Y. Sentoku, S. Akamatsu, W. Sakamoto, R. Kodama, K. A. Tanaka, K. Azumi, T. Norimatsu, T. Matsuoka, H. Fujita, and H. Yoshida, Phys. Rev. Lett. 92, 205002 (2004); F. Dorchies, J. R. Marques, B. Cros, G. Matthieussent, C. Courtois, T. Velikoroussov, P. Audebert, J. P. Geindre, S. Rebibo, G. Hamoniaux, and F. Amiranoff, Phys. Rev. Lett. 82, 4655 (1999).

[9] Y. Ehrlich, C. Cohen, A. Zigler, J. Krall, P. Sprangle, and E. Esarey, Phys. Rev. Lett. 77, 4186 (1996); T. Hosokai, M. Kando, H. Dewa, H. Kotaki, S. Kondo, N. Hasegawa, K. Nakajima, and K. Horioka, Opt. Lett. 25, 10 (2000); A. Butler, D. J. Spence, and S. M. Hooker, Phys. Rev. Lett. 89, 185003 (2002); T. Kameshima, H. Kotaki, M. Kando, I. Daito, K. Kawase, Y. Fukuda, L. M. Chan, T. Homma, S. Kondo, T. Zh. Esirkepov, N. A. Bobrova, P. V. Sasorov, and S. V. Bulanov, Phys. Plasmas 16, 093101 (2009).

[10] C. G. Durfee and H. M. Milchberg, Phys. Rev. Lett. 71, 2409 (1993).

[11] T. R. Clark and H. M. Milchberg. Phys. Rev. E 61, 1954 (2000); T. R. Clark and H. M. Milchberg, Phys. Rev. Lett. 78, 2373 (1997); V. Malka, E. De Wispelaere, F.
Amiranoff, S. Baton, R. Bonadio, C. Coulaud, R. Haroutunian, A. Modena, D. Puissant, C. Stenz, S. Huller, and M. Casanov, Phys. Rev. Lett. 79, 2979 (1997); J. Faure, V. Malka, J.-R. Marques, F. Amiranoff, C. Courtois, Z. Najmudin, K. Krushelnick, M. Salvati, A. E. Dangor, A. Solodov, P. Mora, J.-C. Adam, and A. Heron, Phys. Plasmas 7, 3009 (2000).

[12] K. Krushelnick, A. Ting, C. I. Moore, H. R. Burris, E. Esarey, P. Sprangle, and M. Baine, Phys. Rev. Lett. 78, 4047 (1997); S.-Y. Chen, G. S. Sarkisov, A. Maksimchuk, R. Wagner, and D. Umstadter, Phys. Rev. Lett. 80, 2610 (1998).

[13] A. Giulietti, P. Tomassini, M. Galimberti, D. Giulietti, L. A. Gizzi, P. Koester, L. Labate, T. Ceccotti, P. D'Oliveria, T. Auguste, P. Monot, and P. Martin, Phys. Plasmas 13, 093103 (2006); J. Faure, C. Rechatin, O. Lundh, L. Ammoura, and V. Malka, Phys. Plasmas 17, 083107 (2010).

[14] Ya. B. Zeldovich and Yu.P. Raizer, Physics of Shock Waves and High-Temperature Hydrodynamics Phenomena (Academic Press, New York, 1966), Vols. 1, 2.

[15] M. Mori, A. Pirozhkov, M. Nishiuchi, K. Ogura, A. Sagisaka, Y. Hayashi, S. Orimo, A. Fukumi, Z. Li, M. Kado, and H. Daido, Laser Phys. 16, 1092 (2006).

[16] M. Mori et al., IEEE Trans. Plasma Sci. 36, 1872 (2008).

[17] M. Hipp, J. Woisetschlaeger, P. Reiterer, and T. Neger, Measurement 36, 53 (2004).

[18] M. Takeda, H. Ina, and S. Kobayashi, J. Opt. Soc. Am. 72, 156 (1982).

[19] P. Tomassini and A. Giulietti, Opt. Commun. 199, 143 (2001).

[20] H. Kotaki, M. Kando, T. Oketa, S. Masuda, James K. Koga, S. Kondo, S. Kanazawa, T. Yokoyama, and T. Matoba, Phys. Plasmas 9, 1392 (2002).

[21] A. Zhidkov, T. Esirkepov, T. Fujii, K. Nemoto, J. Koga, and S. V. Bulanov, Phys. Rev. Lett. 103, 215003 (2009); A. Zhidkov, J. Koga, T. Esirkepov, T. Hosokai, M. Uesaka, and T. Tajima, Phys. Rev. E 69, 066408 (2004); A. Zhidkov and A. Sasaki, Phys. Plasmas 7, 1341 (2000).

[22] R. R. Freeman, P.H. Bucksbaum, H. Milchberg, S. Darack, D. Schumacher, and M.E. Geusic, Phys. Rev. Lett. 59, 1092 (1987).

[23] B. B. Pollock, C.E. Clayton, J.E. Ralph, F. Albert, A. Davidson, L. Divol, C. Filip, S. H. Glenzer, K. Herpoldt, W. Lu, K. A. Marsh, J. Meinecke, W. B. Mori, A. Pak, T. C. Rensink, J. S. Ross, J. Shaw, G. R. Tynan, C. Joshi, and D. H. Froula, Phys. Rev. Lett. 107, 045001 (2011); E. Oz et al., Phys. Rev. Lett. 98, 084801 (2007).

[24] S. Kato, Y. Kishimoto, and J. Koga, Phys. Plasmas 5, 292 (1998). 